\title{
Difficult to digest: A hard diagnosis to swallow
}

\section{Justin Lam MD, Laurence Arsenault-Blanchard MD, Alene Toulany MD FRCPC}

Hospital for Sick Children, Toronto, Ontario

Correspondence: Justin Lam, Hospital for Sick Children, 555 University Avenue, Toronto, Ontario M5G 1X8

\section{CASE PRESENTATION}

A 14-year-old female with a 10-year history of Gastroesophageal reflux disease (GERD) and a 1-year history of daily regurgitation was called to the Emergency Department after outpatient bloodwork revealed hypokalemia of $2.5 \mathrm{mmol} / \mathrm{L}$. Over the previous 3 months, she had a $6 \mathrm{~kg}$ weight loss. However, $4.5 \mathrm{~kg}$ of this weight loss occurred in the preceding 3 weeks secondary to an intercurrent illness with upwards of $9 \mathrm{~L}$ of regurgitation per day. At baseline, she reported effortless regurgitation with each meal, with a 9-month history of enteral feeds overnight via self-inserted nasogastric tube for weight stabilization. This was started after initial efforts to increase oral nutrition and decrease symptoms had failed. She endorsed presyncope, but denied palpitations, shortness of breath, blood in her vomit or stool, or any symptoms of inflammatory bowel or rheumatologic disease.

On physical examination, she looked thin and pale. Her heart rate was 67 with a significant orthostatic change of 30 from supine to standing, blood pressure was $105 / 69$, and body mass index was 15.9 (third percentile). The remainder of her examination was unremarkable. Her potassium was $2.7 \mathrm{mmol} / \mathrm{L}$ in the Emergency Room with a normal electrocardiogram. The patient was admitted to the General Paediatrics unit for correction of electrolyte abnormalities and dehydration as well as symptom interruption. 


\section{DISCUSSION}

Given her history of effortless regurgitation and fluctuating weight, she had been diagnosed with rumination syndrome 1 year before this presentation. While in hospital, she had an episode of ventricular tachycardia; a Holter monitor was done and was normal. The Adolescent Medicine team was consulted to rule out a concurrent eating disorder. It became apparent that the patient was worried about not fitting in at her new arts school and had been restricting food for 4 months for fear of regurgitating/vomiting in front of her peers. She also avoided 'leafy' foods because of its texture when regurgitated. She denied body image concerns, fear of gaining weight, or avoiding high energy foods. Given the weight loss and dependence on enteral feeds, a concurrent diagnosis of avoidant restrictive food intake disorder (ARFID) was made.

She was transferred to the Eating Disorders unit for ongoing symptom interruption related to the regurgitation and emesis and medical stabilization with IV fluids and electrolytes. Her emesis improved with continuous enteral feeds via a nasojejunal tube. She continued to take nutrition by mouth as we supported her to try mindfulness techniques and diaphragmatic breathing to decrease her rumination symptoms. She began tolerating oral intake with decreased emesis and was discharged to the outpatient Eating Disorder program for ongoing management when emesis returned to baseline levels (approximately 1-2 L/day).

Rumination syndrome is a functional gastroduodenal disorder which is characterized by persistent or recurrent regurgitation of recently ingested food into the mouth. Although traditionally associated with infants, children, and mentally and developmentally disabled patients, it may also occur in healthy adults, with a predisposition toward females (1). There is often a delay to diagnosis, as rumination often masquerades as other illnesses such as gastroesophageal reflux disease (2).

Rumination episodes are a result of a subconsciously voluntary elevation of intra-gastric pressure that overcomes the lower esophageal sphincter and causes the sphincter to relax. This phenomenon is thought to be triggered by stressful life events or unpleasant abdominal sensations (1). Weight loss in rumination syndrome is common. Rumination syndrome can be distinguished from GERD via $\mathrm{pH}$-impedance monitoring combined with conventional manometry (1).

ARFID is a new diagnosis in the DSM- 5 under Feeding and Eating Disorders. It is characterized by clinically significant struggles with eating and food leading to significant weight loss or growth compromise, dependence on enteral feeding or nutritional supplementation, significant nutritional deficiency, or marked interference with the patient's psychosocial functioning, in the absence of a fear of weight gain, a desire to lose weight, or body image dissatisfaction.

ARFID may also often present with comorbid physical symptoms such as nausea, early satiety, and abdominal pain. A review of the literature revealed only five previous case reports of rumination syndrome with co-existing eating disorders. ARFID can only be diagnosed in situations where the findings cannot be better explained by another eating disorder or medical condition, or, as in this case, if the presentation is out of proportion to what is typically expected of the patient's underlying rumination syndrome (3). Our clinical case highlights that a concurrent eating disorder, such as ARFID, requires a high index of suspicion and should be considered in a patient with rumination syndrome if there is significant weight loss, electrolyte abnormalities, cardiac arrhythmias, significant vital sign instability or orthostatic changes, or a marked change in mood or affect leading to psychosocial dysfunction.

Management of both rumination syndrome and ARFID involves behavioural and medical interventions by a medical and psychiatric inter-disciplinary team (1-3). The treatment of ARFID includes medical stabilization and weight restoration as well as cognitive strategies to address the underlying cause of a patient's eating disturbance (3). The treatment of rumination syndrome is centred on education and often requires behavioural approaches focused on understanding the underlying mechanism and diaphragmatic breathing exercises, with more severe cases requiring more intensive treatment such as habit reversal protocols, structured mealtimes, gastrointestinal pharmacotherapy, and caloric supplementation (2).

\section{CLINICAL PEARLS}

1. Rumination syndrome is a functional gastroduodenal disorder which is characterized by persistent or recurrent voluntary regurgitation of recently ingested food into the mouth.

2. A concurrent eating disorder should be considered in a patient with rumination syndrome when there is weight loss out of proportion to rumination severity, electrolyte abnormalities, cardiac arrhythmias, significant vital sign instability or orthostatic changes, or a change in affect and behaviour.

3. Treatment of rumination syndrome and ARFID involves intensive behaviour and medical management by an inter-disciplinary team.

\section{Potential Conflicts of Interest}

All authors: No reported conflicts of interest. All authors have submitted the ICMJE Form for Disclosure of Potential Conflicts of Interest. Conflicts that the editors consider relevant to the content of the manuscript have been disclosed

Informed consent: The authors received written informed consent from the family to publish this article.

\section{References}

1. Kessing BF, Smout AJ, Bredenoord AJ. Current diagnosis and management of the rumination syndrome. J Clin Gastroenterol 2014;48(6):478-83. 
2. Green $\mathrm{AD}$, Alioto A, Mousa $\mathrm{H}$, Di Lorenzo C. Severe pediatric rumination syndrome: Successful interdisciplinary inpatient management. J Pediatr Gastroenterol Nutr 2011;52(4):414-8.
3. Norris ML, Spettigue WJ, Katzman DK. Update on eating disorders: Current perspectives on avoidant/restrictive food intake disorder in children and youth. Neuropsychiatr Dis Treat 2016;12:213-8. 